\title{
Robust Controllers for Dead-time Systems
}

\author{
Markus Hermle \& Werner Schiehlen \\ Institute B of Mechanics \\ University of Stuttgart \\ 70550 Stuttgart, Germany \\ mh@mechb.uni-stuttgart.de
}

\author{
Hans Zwart \\ Faculty of Applied Mathematics \\ University of Twente \\ 7500 AE Enschede, The Netherlands \\ H.J.Zwart@math.utwente.nl
}

\author{
Ruth Curtain \\ Faculty of Mathematics and Natural Sciences \\ University of Groningen \\ 9700 AV Groningen, The Netherlands \\ r.f.curtain@math.rug.nl
}

\begin{abstract}
It is shown that the classical controllers for dead-time systems, known as Smith predictors, provide a suitable basis for the design of optimal robust stabilizing controllers. Only in case of an unstable plant a modification of the Smith predictor is necessary. A simple first order dead-time system serves as illustrative example.
\end{abstract}

\section{Introduction}

In the late 50s O.J.M. Smith [7], [8] proposed a controller for a process with transportation lag preventing dead-time-exited oscillations. This controller, called Smith predictor, has the unique property that the closed-loop behavior of the system with delay can be obtained via the closed-loop behavior of the delay free part.

The idea of this paper is to show that controllers for dead-time systems using the approach of Curtain and Zhou [1], Curtain and Zwart [2] respectively, for designing robustly stabilizing controllers for infinitedimensional systems can be seen as (modified) Smith predictors. Hence this $H_{\infty}$-controllers for dead-time systems are easy to understand and easy to implement. A modification of the Smith predictor is only necessary if the original plant is unstable.

In Section 2 we present some facts about a class of (irrational) transfer functions and summarize known results on robust control under additive perturbations. The classical Smith predictor is introduced in Section 3. In Section 4 , we define the control problem and show that the robust stabilizing controllers obtained by applying the $H_{\infty}$-approach for dead-time systems are identical with (modified) Smith predictors. In the last section, we apply the derived results to a simple dead-time system.
2 Robust Stability

Although we shall mainly concentrate on transfer functions of the type $g(s) e^{-s T}$, i.e. a cascade of a rational transfer function $g(s)$ and a pure delay $e^{-s T}$ with the dead-time $T>0$, we shall first introduce the general class of irrational transfer functions.

From a systemtheoretic point of view the considered class of irrational transfer functions should form an algebra (see [2, Definition A.7.11]). Moreover, we want to be able to construct feedback configurations which involve the operation of an algebraic inverse. A class of scalar irrational transfer functions that has all these properties is the Callier-Desoer class, which is the topic of this section. We begin by examining the following Banach algebra of impulse responses as discussed in [2, Appendix A.7.4].

Definition 2.1 Let $\beta$ be a real number. We say that $f \in \mathcal{A}(\beta)$ if $f$ is zero on negative time, and has (on positive time) the representation

$$
f(t)=f_{a}(t)+\sum_{n=1}^{\infty} f_{n} \delta\left(t-t_{n}\right),
$$

where $t_{n} \in[0, \infty), f_{n} \in \mathbb{C} ; 0=t_{1}, t_{n}>0$ for $n \geq 2$, $\delta\left(t-t_{n}\right)$ represents the delta distribution centered in $t_{n}, e^{-\beta \cdot} f_{a}(\cdot) \in L_{1}(0, \infty)$ and $\sum_{n=1}^{\infty}\left|f_{n}\right| e^{-\beta t_{n}}<\infty$.

The algebraic properties of $\mathcal{A}(\beta)$ are inherited by the corresponding Laplace transforms and this suggests that $\hat{\mathcal{A}}(\beta)$, the class of Laplace transforms of $\mathcal{A}(\beta)$, should be an appropriate class of $\beta$-exponentially stable irrational transfer functions. While the algebra $\hat{\mathcal{A}}(\beta)$ has exactly the right properties to model a class of stable transfer functions, it has the serious drawback that not all elements in its quotient field have a coprime factorization. Since coprime factorizations play a crucial role in control synthesis of unstable transfer func- 
tions, we introduce two sub-algebras of $\hat{\mathcal{A}}(\beta)$ with which we shall form a quotient algebra with the coprime factorization property. For background material on algebras and $\hat{\mathcal{A}}(\beta)$ see [2, Appendix 7].

Definition 2.2 We define the following sub-algebras of $\hat{\mathcal{A}}(\beta)$ :

a. $\hat{\mathcal{A}}_{-}(\beta):=\left\{\hat{f} \mid \hat{f} \in \hat{\mathcal{A}}\left(\beta_{1}\right)\right.$ for some $\left.\beta_{1}<\beta\right\} ;$

b. Let $\overline{\mathbb{C}_{\beta}^{+}}$denote the set $\{s \in \mathbb{C} \mid \operatorname{Re}(s) \geq \beta\}$.

$$
\begin{aligned}
\hat{\mathcal{A}}_{\infty}(\beta):= & \left\{\hat{f} \in \hat{\mathcal{A}}_{-}(\beta) \mid\right. \text { there exists } \\
& \rho>0 \text { such that } \\
& \left.\underset{\left\{s \in \overline{\mathbb{C}}_{\beta}^{+} \| s \mid \geq \rho\right\}}{\inf }|\hat{f}(s)|>0\right\} .
\end{aligned}
$$

The special property that elements $\hat{f} \in \hat{\mathcal{A}}_{\infty}(\beta)$ have is often expressed as bounded away from zero at infinity in $\overline{\mathbb{C}_{\beta}^{+}}$.

We now define the quotient algebra $\hat{\mathcal{B}}(\beta)$ of $\hat{\mathcal{A}}_{-}(\beta)$ with respect to $\hat{\mathcal{A}}_{\infty}(\beta)$. $\hat{\mathcal{B}}(\beta)$ will represent our class of scalar (possibly unstable) irrational transfer functions.

Definition 2.3 The Callier-Desoer class $\hat{\mathcal{B}}(\beta)$ of transfer functions is defined for any $\beta \in \mathbb{R}$ by

$$
\hat{\mathcal{B}}(\beta):=\hat{\mathcal{A}}_{-}(\beta)\left[\hat{\mathcal{A}}_{\infty}(\beta)\right]^{-1} .
$$

For simplicity of notation, we take $\beta=0$ in the following definition of input-ouput stability.

Definition 2.4 $G \in \hat{\mathcal{B}}(0)$ is said to be input-output stable if $G \in \hat{\mathcal{A}}_{-}(0)$.

By "stabilizing a system $G \in \hat{\mathcal{B}}(0)$ by means of a controller $K \in \hat{\mathcal{B}}(0)$ ", we mean that the closed-loop configuration as given in Figure 1 is stable in the sense that all the paths in the loop are input-output stable.

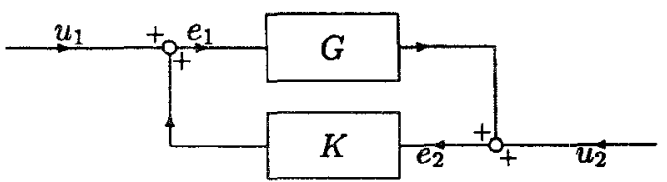

Figure 1: System-controller connection

Calculating the corresponding transfer functions leads to the following definition.

Definition 2.5 The feedback system $(G, K)$ of Figure 1 , where $G, K \in \hat{\mathcal{B}}(0)$, is said to be input-output stable if and only if the transfer functions

$S:=(1-G K)^{-1}, K S$ and $S G$

are in $\hat{\mathcal{A}}_{-}(0), K$ is called a stabilizing controller for $G$. We also say that $K$ stabilizes $G$.
A desirable property of the controller would be that it stabilizes not only the nominal plant, but all plants which are reasonably close to the nominal ones. This type of property is called robust stabilization. There are many types of robustness one can consider, depending on the class of perturbations and the mathematical measure for distance one chooses. In this paper, we consider robustness with respect to additive uncertainty. For more about other classes of uncertainties as well as for the proofs of the quoted results, we refer the reader to Chapter 9 of [2].

Definition 2.6 Let $G$ and $G_{\Delta}$ be transfer functions in $\hat{\mathcal{B}}(0)$ of the nominal and the perturbed plants, respectively. A perturbation $\Delta_{a} \in \hat{\mathcal{B}}(0)$ is an additive uncertainty if

$$
G_{\Delta}=G+\Delta_{a}
$$

In order to prove some theorems about robust stabilization, we need to introduce some further restrictions on the class of perturbations $\Delta_{a}$ allowed.

Definition 2.7 Let $G \in \hat{\mathcal{B}}(0)$. We define $\varepsilon$-admissible perturbations for our class of perturbations as follows: $\Delta_{a} \in \hat{\mathcal{B}}(0)$ is an $\varepsilon$-admissible additive perturbation if

a. it is a strictly proper perturbation with no poles on the imaginary axis, $G$ and $G+\Delta_{a}$ have the same number of unstable poles in $\overline{\mathbb{C}_{0}^{+}}$and $\left\|\Delta_{a}\right\|_{\infty}<\varepsilon$; or

b. it is a stable perturbation with $\left\|\Delta_{a}\right\|_{\infty}<\varepsilon$.

$\sup _{\omega \in \mathbb{R}}\|\Delta(\jmath \omega)\|$ is denoted by $\|\Delta\|_{\infty}$.

If $K \in \hat{\mathcal{B}}(0)$ stabilizes $G+\Delta_{a}$ for all $E$-admissible perturbations, we say that $(G, K)$ is robustly stable with robustness margin $\varepsilon$. We also say that $K$ robustly stabilizes $G$ with robustness margin $\varepsilon$.

In the next theorem, we give necessary and sufficient conditions for robust stability for the uncertainty model in Definition 2.7 .

Theorem 2.8 Suppose that $K, G \in \hat{\mathcal{B}}(0), K \neq 0$, and $G$ is strictly proper on $\overline{\mathbb{C}_{0}^{+}} . K$ stabilizes $G_{\Delta}=G+\Delta_{a}$ for all $\varepsilon$-admissible additive perturbations $\Delta_{a}$ if and only if $K$ stabilizes $G$ and

$$
\left\|K(1-G K)^{-1}\right\|_{\infty} \leq \varepsilon^{-1} .
$$

The question naturally arises as to the largest possible value of $\varepsilon$ one can choose and this depends on the existence of a solution to the $H_{\infty}$-optimization problem

$$
\inf _{K}\left\|K(1-G K)^{-1}\right\|_{\infty}
$$

where $K$ ranges over all controllers which stabilize $G$ in the sense of Definition 2.5. If the $H_{\infty}$-optimization 
problem (4) has an infimum $\gamma_{0}$ and there exists a controller $K_{0}$ such that

$$
\begin{aligned}
& \inf _{K \text { stabilizes } G}\left\|K(1-G K)^{-1}\right\|_{\infty} \\
& =\left\|K_{0}\left(1-G K_{0}\right)^{-1}\right\|_{\infty}=\gamma_{0},
\end{aligned}
$$

then clearly, the maximally achievable value of $\varepsilon$ is $\gamma_{0}^{-1}$. This motivates the following concepts.

Definition 2.9 Suppose that $G \in \hat{\mathcal{B}}(0)$ and that $G$ is strictly proper on $\overline{\mathbb{C}_{0}^{+}}$. If (4) has a solution over the controllers $K \in \hat{\mathcal{B}}(0)$ which stabilize $G$ and there exists a minimizing controller $K_{0} \in \hat{\mathcal{B}}(0)$ satisfying (5) for a $\gamma_{0}<\infty$, then

a. the maximal robustness margin for $G$ is

$$
\begin{aligned}
\varepsilon_{\max } & \\
& :=\gamma_{0}^{-1} \\
& =\left(\inf _{K \text { stab. } G}\left\|K(1-G K)^{-1}\right\|_{\infty}\right)^{-1},
\end{aligned}
$$

b. $K_{0}$ is an optimally robust controller for $G$, with respect to additive perturbations.

If $K$ stabilizes $G$ and $\left\|K(1-G K)^{-1}\right\|_{\infty}=\gamma>\gamma_{0}$, then

c. $(G, K)$ is robustly stable with robustness margin $\varepsilon=\gamma^{-1}$ with respect to additive perturbations.

d. $K$ stabilizes $G$ with robustness margin $\varepsilon=\gamma^{-1}$ with respect to additive perturbations.

\section{Smith predictor}

In this section, we shall introduce a controller design method for dead-time systems, known as the Smith predictor or Smith's control scheme, in reference to O.J.M. Smith, who first published this method in the late $50 \mathrm{~s}$ [7], [8]. The fact that the model of the controlled plant is an explicit part of the controller creates a relatively easy design in familiar delay-free terms.

In the following we derive this control scheme. Let the controller in the delay-free loop of the system, given in Figure 2, be $K(s)$.

The delay-free loop in Figure 2 has the transfer function

$$
H_{c}(s)=\frac{K(s) g(s)}{1-K(s) g(s)} .
$$

Taking the delay-time $T$ into account, the transfer function over the overall system

$$
H_{d}(s)=\frac{K(s) g(s)}{1-K(s) g(s)} \cdot e^{-s T}
$$

has a similar dynamical behavior and differs from $H_{c}(s)$ just by delaying the system output. This is the desired

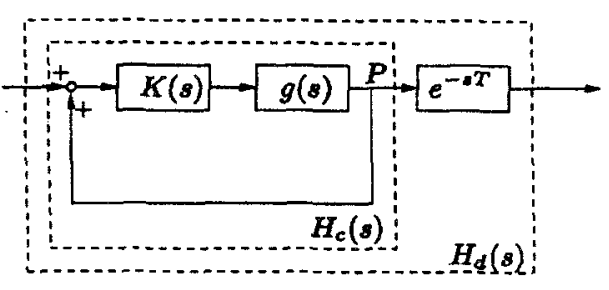

Figure 2: Desired control loop configuration

transfer function using Smith's control scheme. Since the point $P$ is inaccessible in the real plant, the design problem is to relate the controller $\hat{K}(s)$ in Figure 3 to the controller $K(s)$ in Figure 2 such that both systems have identical transfer functions.

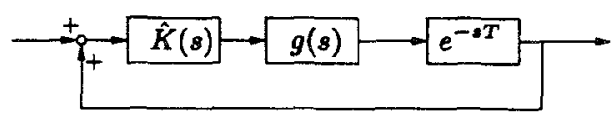

Figure 3: Real control loop configuration

Calculating the transfer function of the real dead-time system from Figure 3 gives

$$
H_{r}(s)=\frac{\hat{K}(s) g(s) \cdot e^{-s T}}{1-\hat{K}(s) g(s) \cdot e^{-s T}}
$$

and by applying the above mentioned condition $H_{r}(s)=$ $H_{d}(s)$, we get

$$
\frac{\hat{K}(s) g(s) \cdot e^{-s T}}{1-\hat{K}(s) g(s) \cdot e^{-s T}}=\frac{K(s) g(s) e^{-s T}}{1-K(s) g(s)} .
$$

After a short computation it follows that

$$
\hat{K}(s)=\frac{K(s)}{1-K(s) g(s)\left(1-e^{-s T}\right)},
$$

which has the physical realization as shown in Figure 4, and which is known as the Smith predictor.

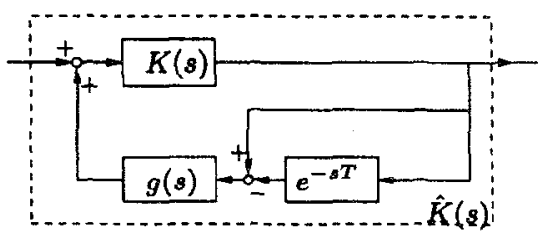

Figure 4: Smith predictor

By using the Smith predictor the dead-time control problem has been reduced to:

1. performing the delay-free design for $(\mathrm{g}, \mathrm{K})$, 
2. realizing the controller $\hat{K}(s)$ (see eqn. (11)).

By implementing the controller $\hat{K}(s)$ as shown in Figure 4 one realizes the desired closed-loop transfer function $H_{d}(s)$ and the stability of the dead-time system can be studied in delay-free terms. This very easy and straightforward way for designing a stable transfer function from the system input to the system output is questionable in case of inaccurate modelling. This fact, mentioned in various books dealing with control engineering, e.g. Föllinger [3], suggests to relate this control scheme with the robust stabilizing controllers introduced in Section 2.

\section{Smith predictors as robust controllers}

Before we can give the relation between robust stabilizing controllers and the Smith predictor, we need the following result which is a simple adaptation of Lemma 2.2 of Curtain and Zhou [1].

Lemma 4.1 Let $G$ be a strictly proper transfer function in $\hat{\mathcal{B}}(0)$ that is written as $G=G_{1}+G_{2}$, with $G_{2}$ in $\hat{\mathcal{A}}_{-}(0)$.

A controller $K \in \hat{\mathcal{B}}(0)$ is a stabilizing controller for $G$ if and only if $K_{1}:=K\left(1-G_{2} K\right)^{-1}$ is a stabilizing controller for $G_{1}$.

Furthermore, the robustness margin of $(G, K)$ is the same as that of $\left(G_{1}, K_{1}\right)$.

Proof: One can prove this by simple algebraic manipulation, as in [1] that extends the proof of Lemma 9.3.1 in [2]. However, we shall present a proof based completely on signal diagrams.

By the definition of $G$ we see that Figure 1 is the same as Figure 5 .

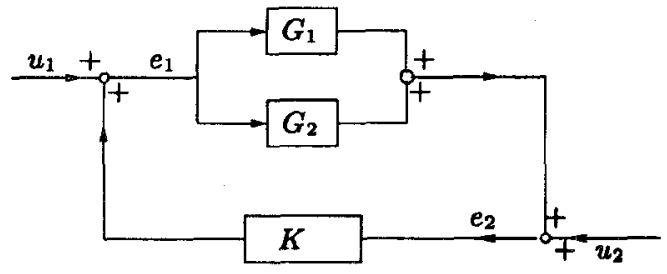

Figure 5: Stabilizing controller, stage 1

Here we can make the two adders on the right-hand side of the figure into one, see Figure 6. Since we want to have just $G_{1}$, we take the part with $G_{2}$ outside the flow diagram as in Figure 7. However, $e_{1}$ is not an input so this has to be changed again. Using that $e_{1}=$ $u_{1}+K e_{2}$, we see that we can change Figure 7 into Figure 8. The transfer function $K_{1}$ of the loop inside the dotted box is $K\left(1-G_{2} K\right)^{-1}$. By the definition of

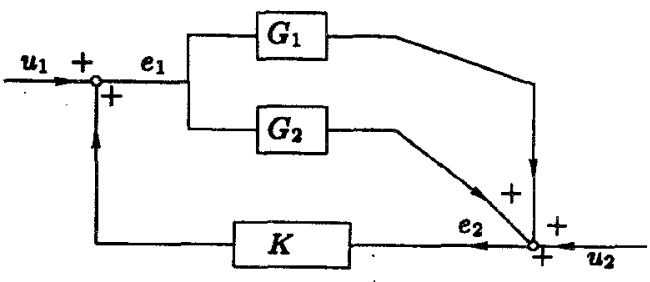

Figure 6: Stabilizing controller, stage 2

stability, we have that the systems in Figure 5 and 8, respectively, are stable if all paths in the loop are inputoutput stable. Since $G_{2}$ is stable, we see that if $K$ is a stabilizing controller for $G$, then $K_{1}$ is a stabilizing controller for $G_{1}$. To get the other implication, one just have to replace in Figure $5 G_{1}$ by $G$ and $G_{2}$ by $-G_{2}$.

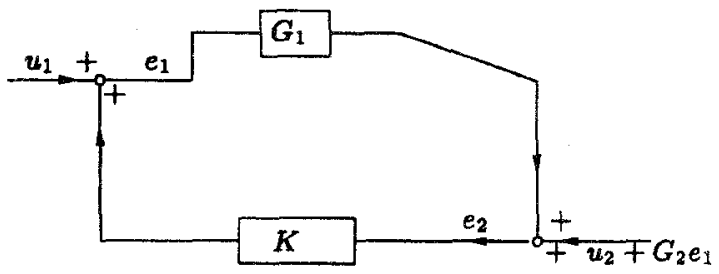

Figure 7: Stabilizing controller, stage 3

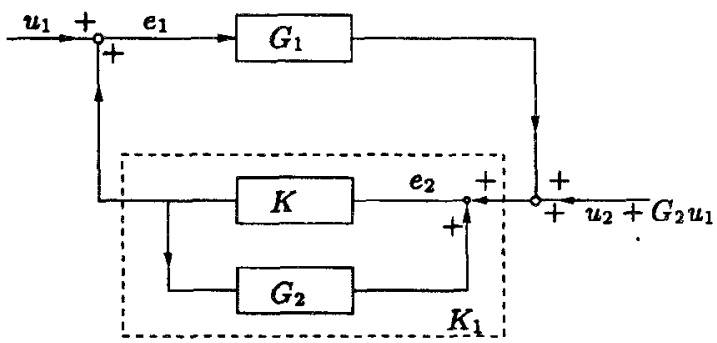

Figure 8: Stabilizing controller, stage 4

From Theorem 2.8, we have that the robustness margin is one over the norm of the transfer function form $u_{2}$ to $e_{1}$, i.e.

$$
e_{1}=K(1-G K)^{-1} u_{2} \text {. }
$$

From Figures 5 and 8 one can easily see that this transfer function between $u_{2}$ and $e_{1}$ remains the same, and so the robustness margins are the same.

From Lemma 4.1 we deduce the following corollary for dead-time systems.

Corollary 4.2 Consider the dead-time system $g(s) e^{-s T}$ with $g$ strictly proper. Let $J$ be a proper (rational) transfer function, such that $g(s)\left[J(s)-e^{-s T}\right]$ 
is stable. If $k$ stabilizes $g J$ with robustness margin $\varepsilon$, then the controller

$$
K_{1}(s):=\frac{k(s)}{1-k(s) g(s)\left[J(s)-e^{-s T}\right]}
$$

stabilizes $g(s) e^{-s T}$ with the same robustness margin.

Proof: Take in Lemma 4.1 $G(s)=g(s) J(s), G_{1}(s)=$ $g(s) e^{-s T}$, and $G_{2}(s)=g(s)\left[J(s)-e^{-s T}\right]$. Then we see that $G_{2}$ is stable, and if $k$ stabilizes $G$ then $K_{1}(s):=$ $k(s)\left(1-k(s) g(s)\left[J(s)-e^{-s T}\right]\right)^{-1}$ stabilizes $G_{1}(s)=$ $g(s) e^{-s T}$.

We see that the controller defined by (13) can be realized as in Figure 9. Notice, that in case of a stable

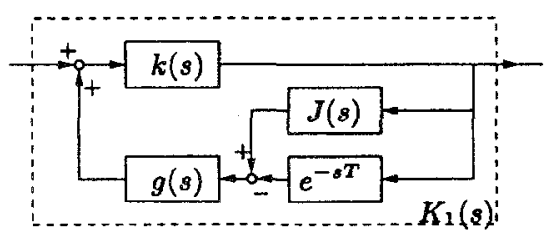

Figure 9: Modified Smith predictor

$g(s)$ in Corollary $4.2 J(s)$ can be any stable and proper transfer function. If we choose $J(s)=1$ we obtain the Smith predictor introduced in Section 3.

Motivated by this similarity to the Smith predictor, we call this controller the modified Smith predictor. We shall apply this controller to a simple (unstable) deadtime system.

\section{First order dead-time systems}

In order to illustrate the results of the previous sections, we consider an unstable dead-time system of first order. Using the results in Glover [4], one can calculate the optimal robust controller for these systems. We shall derive this optimal robust controller using the conventional method of loop shaping. The numerical calculations for all examples in this section have been executed using MATLAB [6]. More detail can be found in Hermle [5].

The dead-time system that we consider is given by

$$
G_{1}(s)=\frac{e^{-s T}}{s+q}
$$

where $T>0$ and $q<0$.

From [2, Example 9.3.5] we know that the maximal achievable robustness margin for this dead-time system is given by $\varepsilon_{\max }=\frac{e^{q T}}{-2 q}$. Using Corollary 4.2, we want to design a controller with this (maximal) robustness margin. First we have to find a rational $J$ such that

$$
G_{2}(s)=\frac{J(s)-e^{-s T}}{s+q}
$$

is stable. Easily one sees that with the constant

$$
J=e^{q T},
$$

this will hold. With this, the "auxiliary" system without dead-time for which we have to find a controller is given by

$$
G(s)=g(s) J=\frac{e^{q T}}{s+q} .
$$

A. controller design by loop-shaping means above all that we have to find a stabilizing controller. Beside stability loop-shaping provides some possibilities to realize further desirable properties of the closed-loop system, like robustness and performance. The graphical means of the loop-shaping method are the Nyquist and Bode plots of the open-loop.

The Nyquist criterion uses the Nyquist plot of the open-loop $c(s) g(s)$ in Figure 10 in order to determine

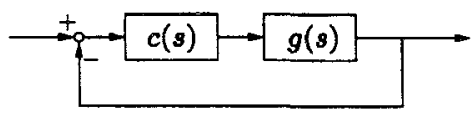

Figure 10: Control loop for the Nyquist criterion

if a certain controller $c(s)$ stabilizes a plant $g(s)$.

Theorem 5.1 Nyquist criterion. Let $c(s)$ and $g(s)$ be real, rational transfer functions, having $\gamma_{c}, \gamma_{g}$ poles in $\mathbb{C}_{0}^{+}$, respectively, and none on the imaginary axis. Suppose furthermore, that none of these poles is cancelled in the cascade connection $c(s) g(s)$. Then $c(s)$ stabilizes $g(s)$ if and only if the plot of $c(j \omega) g(j \omega)$ as $\omega$ increases from $-\infty$ to $\infty$ does not pass through the point $(-1, j 0)$, and encircles $(-1, j 0) \gamma_{c}+\gamma_{g}$ times in the counter-clockwise sense.

Let us do a design for $q=-1$ and $T=0.25$ :

$$
g(s) J=\frac{e^{-0.25}}{s-1}
$$

The Nyquist plot of (18) is shown in Figure 11. The simplest statement for a stabilizing controller for the "auxiliary" system $g(s) J$ is a P-controller

$$
C(s)=P \text {. }
$$

Since the number of unstable poles $\gamma_{g J}=1$, the plot of $P g(j \omega) J$ has to encircle the point $(-1, j 0)$ once in the counter-clockwise sense as $\omega$ increases from $-\infty$ to $\infty$. Then, according to the Nyquist criterion 5.1, the closed-loop configuration as shown in Figure 10 is stable. The lower bound $P_{\min }$ for $P$ to provide this encirclement is easily found with

$$
P_{\min } g(0) J=-1
$$




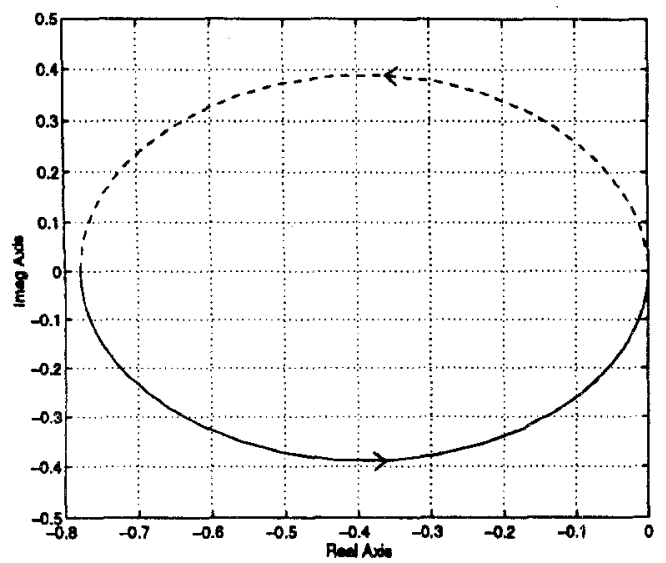

Figure 11: Nyquist plot of $g(s) J$

Therefore,

$$
P>P_{\min }=e^{0.25},
$$

in order to provide stability of the closed-loop system. The robustness properties of the delay-free controller design will be preserved for the dead-time system by using the modified Smith predictor, according to $\mathrm{Co}$ rollary 4.2 In the engineering terminology the Nyquist plot gives an indication of robustness by the distance between the Nyquist plot and the point $(-1, j 0)$. In order to obtain robustness, $P g(j \omega) J$ has to be "as far as possible" from the crucial point $(-1, j 0)$ for all $\omega$. This can be done by choosing

$$
P g(0) J=-2,
$$

in order to draw a circle around $(-1, j 0)$.

With this, $P=2 e^{0.25}$ or for the general case

$$
P=-\frac{2 q}{J}=-2 q e^{-q T} \text {. }
$$

The corresponding Nyquist plot (Figure 12) of $P g(j \omega) J$ shows that the closed-loop system is stable according to the Nyquist criterion 5.1, since the curve encircles the point $(-1, j 0)$ once in the counter-clockwise sense. Since in the control loop for the Nyquist criterium, we have negative feedback, whereas in the rest of this paper we have positive feedback, we have that the $k$ in Corollary 4.2 is $-P$. The calculation of the achieved robustness margin $\varepsilon$ with respect to additive perturbations for this controller using equation (3) yields (in the general case)

$$
\varepsilon=\frac{e^{q T}}{-2 q}
$$

which equals the maximal robustness margin. Hence the modified Smith predictor (13)

$$
K_{1}(s)=\frac{-P}{1+P g(s)\left(J-e^{-s T}\right)},
$$

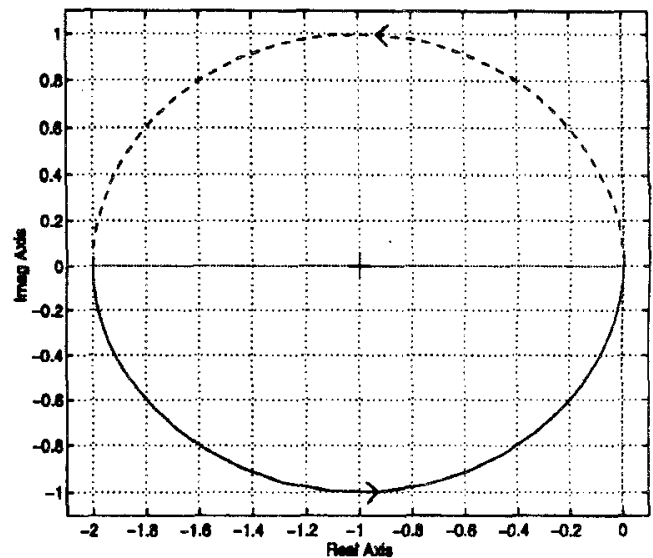

Figure 12: Nyquist plot of $P g(s) J$

with,

$$
\begin{aligned}
& P=-2 q e^{-q T}, \\
& J=e^{q T},
\end{aligned}
$$

is the optimally robust controller for the dead-time system $G_{1}(s)$ from equation (14).

\section{References}

[1] Curtain, R.F. and Zhou, Y., 1994, A Weighted Mixed-Sensitivity $H_{\infty}$-Control Design for irrational Transfer Matrices, IEEE Trans. Automat. Contr. vol. 41, pp. 1312-1321, 1996.

[2] Curtain, R.F. and Zwart, H.J., 1995, An Introduction to Infinite-Dimensional Linear Systems Theory, TAM-21, Springer Verlag, New York.

[3] Föllinger, O., 1992, Regelungstechnik - Einführung in die Methoden und ihre Anwendung, Hüthig, Heidelberg.

[4] K. Glover, K., 1986, Robust Stabilization of Multivariable Linear Systems: Relations to Approximation, Int. J. Control, 43, pp. 741-766.

[5] Hermle, M., 1996, Robust Control of Unstable Dead-Time Systems, Diplomarbeit, University of Stuttgart, ISR, Germany.

[6] MATLAB, developed and copyright by The MathWorks Inc., Natick, Mass., 1994.

[7] Smith, O.J.M., 1957, Closed control of loops with dead-time. Chem. Eng. Progress, 53(5), pp. 217-219.

[8] Smith, O.J.M., 1959, A controller to overcome dead-time. ISA Journal, 6, pp. 28-33. 